\title{
Predicting long-term multicategory cause of death in patients with prostate cancer: random forest versus multinomial model
}

\begin{abstract}
Jianwei Wang, MD, PhD; ${ }^{1}$ Fei Deng, PhD; ${ }^{2}$ Fuqing Zeng, MD; ${ }^{3}$ Andrew J. Shanahan, MD, FACC; ${ }^{4}$ Wei V. Li, ${ }^{5}$ Lanjing Zhang, MD, FRCPath ${ }^{6-8}$
${ }^{1}$ Department of Urology, Beijing Jishuitan Hospital, the Fourth Medical College of Peking University, Beijing, China; ${ }^{2}$ School of Electrical and Electronic Engineering, Shanghai Institute of Technology, Shanghai, China; ${ }^{3}$ Department of Urology, Wuhan Union Hospital of Tongji Medical Collage, Huazhong University of Science and Technology, Wuhan, China; ${ }^{4}$ Department of Medicine, Princeton Medical Center, Plainsboro, NJ; ${ }^{5}$ Department of Biostatistics and Epidemiology Rutgers School of Public Health, Piscataway, NJ; ${ }^{6}$ Department of Pathology, Princeton Medical Center, Plainsboro, NJ; ${ }^{7}$ Department of Biological Sciences, Rutgers University, Newark, NJ; ${ }^{8}$ Rutgers Cancer Institute of New Jersey, New Brunswick, NJ; ${ }^{9}$ Department of Chemical Biology, Ernest Mario School of Pharmacy, Rutgers University, Piscataway, NJ.
\end{abstract}

Correspondence: Lanjing Zhang, MD, Department of Pathology, Princeton Medical Center, 1 Plainsboro Rd, Plainsboro, NJ 08536, USA. Tel: 609853-6833, Tax:609-853-6841, Emails: lanjing.zhang@rutgers.edu or ljzhang@hotmail.com

Running head: Random forest model for predicting cause of death

Key words: Prostate cancer, cause-specific mortality, machine learning, prediction, prognosis

\section{Abstract}

Patients with prostate cancer more likely die of non-cancer cause of death (COD) than prostate cancer. It is thus important to accurately predict multi-category COD in these patients. Random forest (RF), a popular machine learning model, has been shown useful for predicting binary cancer-specific deaths. However, its accuracy for predicting multi-category COD in cancer patients is unclear. We included patients in Surveillance, Epidemiology, and End Results-18 cancer registry-program with prostate cancer diagnosed in 2004 (followed-up through 2016). They were randomly divided into training and testing sets with equal sizes. We evaluated prediction accuracies of RF and conventional-statistical/multinomial models for 6-category COD by data-encoding types using the 2-fold cross-validation approach. Among 49,864 prostate cancer patients, $29,611(59.4 \%)$ were alive at the end of follow-up, and 5,448 (10.9\%) died of cardiovascular disease, 4,607 (9.2\%) of prostate cancer, 3,681 (7.4\%) of Non-Prostate cancer, $717(1.4 \%)$ of infection, and 5,800 (11.6\%) of other causes. We predicted 6-category COD among these patients with a mean accuracy of $59.1 \%(n=240,95 \% \mathrm{Cl}, 58.7 \%-59.4 \%)$ in RF models with one-hot encoding, and $50.4 \%$ (95\% Cl, 49.7\%-51.0\%) in multinomial models. Tumor characteristics, prostatespecific antigen level, and diagnosis confirmation-method were important in RF and multinomial models. In RF models, no statistical differences were found between the accuracies of development versus cross validation phases, and those of categorical versus one-hot encoding. We here report a RF model that has an accuracy of $59.1 \%$ in predicting long-term 6-category COD among prostate cancer patients. It outperforms multinomial logistic models (absolute prediction-accuracy difference, $8.7 \%$ ).

\section{Introduction:}

Prostate cancer is the most prevalent cancer and the second leading-cause of cancer deaths among men in the U.S.A., accounting for 174,650 new cases and 31,620 deaths in 2019.[1, 2] More patients with prostate cancer died of non-cancer causes than of prostate cancer.[3, 4] It is thus important to understand, predict and prevent non-cancer causes of death (CODs) among these patients, particularly cardiovascular disease (CVD).[5] However, only a limited number of studies investigated multicategory COD in prostate cancer patients, and none of them were focused on the prediction of COD. $[3,5,6]$

The random forest (RF) model, a widely-used machine/statistical learning model, improves the performance of decision trees through 
random sampling of training data when building trees and random subsetting of features when splitting nodes.[7] The RF model often outperforms several machine learning and conventional statistical (e.g. logistic regression) models in predicting binary cancer-specific or all-cause deaths, [8-12] with exceptions in a few simulation or biomarker studies.[13, 14] It has also been used to predict cancer-specific deaths in prostate cancer patients.[15] However, few studies have used RF model for predicting multi-category COD in cancer patients, or compared the prediction accuracies of RF versus conventional statistical model (e.g. multinomial logistic regression) for multicategory COD. Our research aims to fill this gap, and we designed a population-based observational study to predict 12-year multicategory COD in prostate cancer patients using RF and multinomial logistic models.

\section{Methods:}

\section{Patient Data}

We extracted individual-patient data from the Surveillance, Epidemiology, and End Results-18 (SEER-18) Program (www.seer.cancer.gov) SEER*Stat Database with Treatment Data using the SEER*Stat software (Surveillance Research Program, National Cancer Institute SEER*Stat software (seer.cancer.gov/seerstat) version $<8.3 .6>$ ).[16] SEER-18 is the largest SEER database including cases from 18 states and covers near $30 \%$ of the U.S. population.[17] The datasets have been widely used and validated for research on breast and colorectal cancers.[18-20] Any summary data involving fewer than 15 patients were statistically suppressed to protect patient identity. Since the SEER database is an existing, de-identified and publicly available dataset, this study is exempt from Institutional Review Board (IRB) review under exempt category 4.

We included all qualified invasive prostate cancer cases in SEER-18 diagnosed in 2004
(2019 data-release, followed up through December 2016). The diagnosis year of 2004 was chosen because the $6^{\text {th }}$ edition of the Tumor, Node and Metastasis staging manual (TNM6) of the American Joint Commission on Cancer (AJCC) was started in 2004 and allowed 12 years of follow-up. But, the AJCC $7^{\text {th }}$ edition of the Tumor, Node and Metastasis staging manual (TNM7) was started in 2010, and would allow only up to 6 years of follow-up, which was not long enough in our view. The inclusion criteria were survival time longer than 1 month, aged 20 years and older, with known COD and first primary only.

\section{Outcome and Variables}

The outcome of the statistical models was the patients' 6-category COD. The COD were originally classified using SEER's recodes of the causes of death according to the COD definition of the U.S. Mortality Data, which were extracted from underlying cause of death on the death certificates of deceased patients.[21] The underlying COD was the unique and most important etiology of the patients' death, while other causes may link to the death and be recorded as other COD on the death certificate. We simplified the SEER COD into 6 categories based on the prevalence of $C O D,[3,6,15]$ including alive, CVD, infection, non-prostate cancer, prostate cancer and others.

The following factors were included in the analysis as variables in RF or multinomial models: age at diagnosis, race/ethnicity (nonHispanic White, Hispanic, non-Hispanic Black, Asian and Pacific Islanders, and others),[22] T, N and $M$ categories of TNM6, AJCC TNM6 clinical staging, prostate specific antigen level (PSA, $\mathrm{ng} / \mathrm{ml}$ ), sum of the Gleason score, chemotherapy, radiotherapy, surgery, and attributes of the county where the patient resided at the time of diagnosis.[23] The PSA levels and Gleason scores were collected from medical records as site specific factors of 
prostate cancer since 2010.[24, 25] Specifically, sums of the Gleason score were obtained from pathology report of resected specimen when available, or that of biopsy specimen if no surgery done. The 4 census-regions of patient's residence county were defined by the U.S. Census Bureau.[26] We converted continuous variables into 4-category variables based on their quartiles. The chemotherapy and radiotherapy data were obtained after signing a user agreement.[25, 27] It is noteworthy that no or unknown status of these treatments should be considered less reliable, while receipt of these treatments was generally confirmed and reliable. $[25,27]$

\section{Statistical analysis}

We compared the accuracies of the RF and multinomial logit models after tuning the parameters of the RF model and choosing the model with the best accuracy. Using the twofold cross-validation approach, the patients were first randomly divided into two subsets of similar sizes ( $n=25,000$ and 24,864, respectively). In each round of the validation, one subset is treated as the training data for constructing models, and the other subset is treated as the test data for evaluating prediction performance (Figure 1). For data-quality assurance, we compared the covariates in the training and testing sets using Chi-square or Student's $t$ test. We identified the RF model with the best accuracy, which is termed as tuning process in data science. Specifically, we examined prediction accuracies (i.e. 1 - classification error) of the models with various numbers of iterations (from 50 to 800 by an interval of 50 ) and variables (from 1 to 15), which were the number of computation rounds and the preset number of the features in RF model, respectively. After the two rounds of validation, the set of parameters that led to the RF model with the smallest average classification error were selected. This cross-validation process is outlined in Figure 1.
Several sensitivity analyses were performed on RF models. To exclude patients lost to follow-up, we conducted training and validation processes in the patients who died during the follow-up or was alive for $>150$ months ( 12.5 years). We also generated training and testing sets with balanced distribution in all tested independent and dependent variables, which were assessed using Chi-square or Student's $t$ test by each variable.

A previous study has shown that one-hot encoding could sometimes outperform complex encoding systems.[28] This approach was also used in machine learning models of cancer driver genes.[29] For one-hot encoding, all multicategory variables (i.e. discrete variables with more than two catrgories) were transformed into a new set of binary variables. For example, the categorical variable for race/ethnicity group would be replaced by 5 binary variables representing whether the patients are non-Hispanic White, Hispanic, nonHispanic Black, Asian and Pacific Islanders, or others, respectively. We trained the RF or multinomial logit models using the one-hot encoded data, and compared the results with those using multicategory variables.

For the multinomial logistic regression model, we first constructed the model using the training set (the subset with sample size of 25,000 ) and predicted the 6-category COD using testing set (Figure 1). If the predicted probability of a given COD was higher than 0.5 , the COD would be assigned to the COD of the patient. Ideally, only one COD had a predicted probability $>0.5$ and was allowed for each case, thus any patient with 0 or $>1$ predicted COD was considered unsuccessfully predicted using multinomial model.

We carried out the above statistical analyses using the RF package and multinomial logistic models of Stata (version 16, College Station, TX).[30-32] The 95\% confidence intervals (CI) of prediction accuracies were estimated using both binomial and Poisson models, that 
produced very similar results. All $P$ values were two-tailed, and a $P$ value $<0.05$ was considered statistically significant.

\section{Results:}

\section{Patients}

We identified and analyzed 49,864 men with prostate cancer diagnosed in 2004 in the SEER18 (Table 1), including 29,611 (59.4\%) alive, 5,448 (10.9) died of CVD, 4,607 (9.2\%) of prostate cancer, 3,681 (7.4\%) of non-prostate cancer, 717 (1.4\%) of infection, and 5,800 (11.6\%) of other causes. The mean survival time was 117 months, while there were 31,273 patients who died during followup or was alive for $>150$ months. Majority of the cancers were of AJCC 6 stage $2(80.9 \%)$ and not treated with prostatectomy (61.6\%). We randomly divided the cases into training and testing sets (STable 1), and found the outcome and all covariates were similarly distributed in these sets, except radiotherapy status ( $P=0.047)$. We then sorted the data by outcome and radiotherapy, randomized the cases again, and achieved similar distributions of the outcome and on covariates in the two sets (STable 2). For the sensitivity analyses on the patients who died during followup or was alive $>150$ months, CODs were similarly distributed in the training and testing sets (Stable 3).

\section{Predicting multi-category causes of death with random forests model}

There were 17 variables with categorical encoding and 61 variables with one-hot encoding, and 240 candidate models in each tuning process. Our tuning processes showed that the prediction accuracy increased with the iteration number in either conventionally or one-hot encoded data (Figure 2), as shown before.[30] The mean prediction-accuracy for 6category COD were $58.6 \%$ (95\% Cl, 58.2\%-59.1\%) in the RF models with conventional encoding and $59.1 \%(95 \% \mathrm{Cl}, 58.7 \%-59.4 \%)$ in those with one-hot encoding. The best accuracy was reached in the model of 3 variables and 800 iterations with conventional encoding (59.2\%, 95\% Cl [58.6\%-59.8\%], Table 2 and Figure 3) and that of 1 variable and 700 iterations with one-hot encoding (59.6\%, 95\% Cl [58.9\%-60.2\%], STable 4 and Figure 3). The best RF model with one-hot encoding appeared to outperform that with conventional encoding, but no statistically significant difference was found. Alive was the COD that all RF models could predict with the best accuracy, while cancer pathological staging and age at diagnosis were top-important factors in the RF models (Figure $\mathbf{3}$ ).

The sensitivity analyses revealed that the prediction accuracies were statistically similar in the -training phase, and cross-validation phase, but statistically lower in the models in patients who died during follow-up or was alive for $>150$ months (Figure 3).

\section{Predicting multi-category causes of death with multinomial model}

As the RF models, and the multinomial logistic regression models with one-hot encoding seemed to have better goodness of fit than with categorical encoding (Pseudo/adjusted Rsquare $=0.1707$ versus 0.1416 , Likelihood Ratio [Chi-square] 10854.51 vs 9009.2, respectively). Because multinomial models used a ranking approach to determine the best-fit outcome, it is possible that more than one outcome (i.e. COD) had a probability $>0.5$. However, the predicted COD in multinomial model was only unique in being alive among the 6-category COD and all other categories were of $<0.5$ probability (Table 2 and STable 4). The mean prediction-accuracy was $50.4 \%(95 \% \mathrm{Cl}, 49.7 \%$ $51.0 \%$ ) in the multinomial models, and lower than RF models, except the RF model on the patients who died during followup or was alive for $>150$ months (Figure 3). Age at diagnosis, AJCC6 staging, confirmation method of diagnosis, surgery and PSA level were 
associated with all 6-category COD in multinomial model, while other factors were only linked to some of the 6-category COD (STable 5).

\section{Discussion}

In this study, we investigated the multilevel prediction problem of prostate patients' COD using a carefully constructed and rigorously tuned RF model. In the patients with prostate cancer diagnosed in 2004, 59.4\% were alive at the end of 12-year follow- up, while the top-3 CODs were CVD, prostate cancer and nonprostate cancer. We predicted 6-category COD among these patients with a mean accuracy of $59.1 \%$ (95\% Cl, 58.7\%-59.4\%) in the tuned RF model with one-hot encoding, and 50.4\% (95\% $\mathrm{Cl}, 49.7 \%-51.0 \%)$ in the multinomial logit model, suggesting RF models outperformed multinomial model. Tumor characteristics, PSA level, diagnosis confirmation-method, and radiotherapy status were the top-ranked variables in RF model, but only age, surgery, diagnosis confirmation-method, PSA level and AJCC6 6 stages as the factors were linked to all of the COD (versus alive) in multinomial models.

The proportions of various COD in our study are similar to those in previous reports.[4] Given the increasing proportion of deaths from COD other than prostate cancer, it is critical to accurately predict or identify the factors linked to these COD among prostate cancer patients. Several studies have attempted to predict cancer-specific or all-cause deaths in prostate cancer patients using clinical pathological and genomic/genetic factors.[15, 33-36] However, few studies to our knowledge predict the causes of death in multiple categories. Multinomial logistic regression is suitable for analyzing categorical/multi-category outcomes.[31, 32] In this study, multinomial logistic regression seems only able to predict the alive status of the 6-category COD if a unique COD successfully identified. In the meantime, a tuned RF model outperformed multinomial logistic regression in predicting 6category COD by $17.2 \%$ higher prediction accuracy ( $8.7 \%$ absolute accuracy-difference). This finding supports that RF's accuracy is similar to or better than support vector machines, artificial neural network and logistic regression in predicting various clinical outcomes, $[9-11,37]$ but contrasts to that its accuracy is inferior to that of logistic regression.[38] It is plausible, but needs additional validation, that RF could also be highly useful in predicting multi-category COD or outcomes of other diseases. Despite the slightly better accuracy linked to data with onehot encoding than standard encoding, we found no statistical differences between the two methods. This finding is inconsistent with previous reports, $[28,29]$ and needs further validation. We also noticed that the minimal depths of trees in our best-fit RF models were usually 1 to 3 . Those observations may help develop and improve machine learning models for predicting multi-category COD in cancer or other patients.

Some of this study's strengths are noteworthy. First, this population-based study provides early evidence on the frequencies of various COD among the prostate cancer patients who were followed up for 12 years. Second, we tuned RF models for predicting 6-category COD in prostate cancer patients, while existing RF models on prostate cancer only predicted binary cancer-specific death, [15, 33] all-cause death, [33, 39] or cancer recurrence.[40] Compared with binary death-outcomes, multiple-category COD are more informative, but more difficult to predict. This is supported by the low success rate of multinomial models in predicting unique COD. Third, the tuned RF models in this study outperformed multinomial models in predicting 6-category COD. Indeed, the multinomial model was only able to predict alive as a unique COD, and missed other COD. Fourth, we characterized RF models and identified the model with best accuracy, while 
few of the prior works tuned their models.[15, $33,40]$ Fifth, we are able to able to achieve a promising prediction accuracy given the large sample-size of this prostate cancer dataset and the cross-validation.[41] Some of prior studies on prostate cancer survivals using machine learning/RF model had either large sample size[15] or cross validation,[42-44] but few combined both. Small sample size was indeed reported as the most common limitation of machine learning studies on cancer prognosis and prediction.[41] Finally, we identified several factors linked to long-term 6-category COD in prostate cancer patients, including age, PSA level and tumor characteristics, as shown by both RF and multinomial models.

This study has the following limitations. The prediction accuracy for 6-category COD in this study is not yet as good as prediction for binary outcomes, such as all-cause deaths.[33] Moreover, despite some shared linked-factors, $\mathrm{RF}$ models did not completely agree with multinomial models on the factors linked to 6category COD. However, RF and other machine learning models are known for their limitations in identifying associated factors.[45] In addition, an outside validation dataset might be needed, but unavailable, largely due to the lack of registry-data. SEER18 is the largest population cancer dataset in the North America.[16] Thus, it is very challenging to obtain another population dataset of similar size for validation. However, we prospectively used the crossvalidation approach to validate our findings, as recommended.[41, 45] Finally, Gleason scores were available in a very small proportion of the patients, but might otherwise improve prediction accuracy.[46]

\section{Conclusions}

In this population-based study, CVD, prostate cancer and non-prostate cancer were the most common long-term COD among prostate cancer patients. RF and multinomial models could predict 6-category COD among these patients with acceptable prediction accuracy, which needs improvement. Those models enable clinicians to gain more granular prognostic information on prostate cancer patients, and target at relevant COD to improve survival. We also show that a tuned RF model outperforms multinomial models by $8.7 \%$ (absolute difference), or 15,195 person-case for the cases diagnosed in 2019 alone in the U.S. Additional works are needed to better predict multiplecategory COD of other cancers.

\author{
Statement \\ - Availability of data and material (Mandatory): \\ All data are available through SEER after signing \\ an agreement. \\ - Competing interests: None to declare. \\ - Funding: None. \\ - Authors' contributions: JW, FZ, AJS and LZ \\ designed the study, JW, FD and LZ extracted \\ and analyzed the data, JW and LZ wrote the first \\ draft of the manuscript and all authors edited \\ the manuscript. The final manuscript was \\ approved by all authors except FZ, who \\ unfortunately passed away on Oct. 1, 2018.
}

\section{Key Points:}

- A tuned random forest model could reach an accuracy of $59.1 \%$ in predicting long-term 6category cause of death among U.S. prostate cancer patients.

- Random forest model outperforms conventional-statistical/multinomial models by an absolute percent difference of $8.7 \%$, but its accuracies did not differ by data coding (conventional versus one-hot encoding). 
bioRxiv preprint doi: https://doi.org/10.1101/2020.01.03.893966; this version posted March 6, 2020. The copyright holder for this preprint (which was not certified by peer review) is the author/funder, who has granted bioRxiv a license to display the preprint in perpetuity. It is made available under aCC-BY-NC-ND 4.0 International license.

- Tumor characteristics, prostate-specific antigen level, and diagnosis confirmation-method appear important for predicting 13-year 6-category cause of death in both random forest and multinomial models. 


\section{Bibliography}

1. Siegel RL, Miller KD, Jemal A: Cancer statistics, 2019. CA Cancer J Clin 2019, 69(1):7-34.

2. Miller KD, Nogueira L, Mariotto AB, Rowland JH, Yabroff KR, Alfano CM, Jemal A, Kramer JL, Siegel RL: Cancer treatment and survivorship statistics, 2019. CA Cancer J Clin 2019, 69(5):363385.

3. Zaorsky NG, Churilla TM, Egleston BL, Fisher SG, Ridge JA, Horwitz EM, Meyer JE: Causes of death among cancer patients. Annals of oncology : official journal of the European Society for Medical Oncology 2017, 28(2):400-407.

4. Epstein MM, Edgren G, Rider JR, Mucci $\mathrm{LA}$, Adami HO: Temporal trends in cause of death among Swedish and US men with prostate cancer. J Natl Cancer Inst 2012, 104(17):1335-1342.

5. Walter SD, de Koning HJ, Hugosson J, Talala K, Roobol MJ, Carlsson S, Zappa M, Nelen V, Kwiatkowski M, Paez A et al: Impact of cause of death adjudication on the results of the European prostate cancer screening trial. Br J Cancer 2017, 116(1):141-148.

6. Nguyen-Nielsen $\mathrm{M}$, Moller $\mathrm{H}$, Tjonneland $\mathrm{A}$, Borre $\mathrm{M}$ : Causes of death in men with prostate cancer: Results from the Danish Prostate Cancer Registry (DAPROCAdata). Cancer Epidemiol 2019, 59:249-257.

7. Breiman L: Random Forests. Machine Learning 2001, 45(1):5-32.

8. Sakr S, Elshawi R, Ahmed AM, Qureshi WT, Brawner CA, Keteyian SJ, Blaha MJ, Al-Mallah $\mathrm{MH}$ : Comparison of machine learning techniques to predict all-cause mortality using fitness data: the Henry ford exerclse testing (FIT) project. BMC medical informatics and decision making 2017, 17(1):174.
9. Peng SY, Chuang YC, Kang TW, Tseng KH: Random forest can predict 30-day mortality of spontaneous intracerebral hemorrhage with remarkable discrimination. European journal of neurology 2010, 17(7):945-950.

10. Shi M, He J: SNRFCB: sub-network based random forest classifier for predicting chemotherapy benefit on survival for cancer treatment. Molecular bioSystems 2016, 12(4):12141223.

11. Montazeri M, Montazeri M, Montazeri $M$, Beigzadeh A: Machine learning models in breast cancer survival prediction. Technology and health care : official journal of the European Society for Engineering and Medicine 2016, 24(1):31-42.

12. Bartholomai JA, Frieboes HB: Lung Cancer Survival Prediction via Machine Learning Regression, Classification, and Statistical Techniques. Proceedings of the IEEE International Symposium on Signal Processing and Information Technology IEEE International Symposium on Signal Processing and Information Technology 2018, 2018:632-637.

13. van der Ploeg T, Austin PC, Steyerberg EW: Modern modelling techniques are data hungry: a simulation study for predicting dichotomous endpoints. $B M C$ medical research methodology 2014, 14:137.

14. Kim S, Park $\mathrm{T}$, Kon $\mathrm{M}$ : Cancer survival classification using integrated data sets and intermediate information. Artificial intelligence in medicine 2014, 62(1):2331.

15. Hanson HA, Martin C, O'Neil B, Leiser CL, Mayer EN, Smith KR, Lowrance WT: The Relative Importance of Race Compared to Health Care and Social Factors in Predicting Prostate Cancer Mortality: A Random Forest Approach. The Journal 
of urology

2019:101097ju0000000000000416.

16. Surveillance $\mathrm{E}$, and End Results (SEER)

Program (www.seer.cancer.gov):

SEER*Stat Database: Incidence - SEER

18 Regs Research Data, Nov 2018 Sub

(1975-2016) <Katrina/Rita Population

Adjustment> - Linked To County

Attributes - Total U.S., 1969-2017

Counties, National Cancer Institute, DCCPS, based on the November 2018 submission. In.; 2019.

17. Number of Persons by Race and Hispanic Ethnicity for SEER Participants (2010 Census Data)

[https://web.archive.org/web/2019102 8021627/https://seer.cancer.gov/regist ries/data.html]

18. Chavali LB, Llanos AAM, Yun JP, Hill SM, Tan XL, Zhang L: Radiotherapy for Patients With Resected Tumor DepositPositive Colorectal Cancer: A

Surveillance, Epidemiology, and End Results-Based Population Study. Arch Pathol Lab Med 2018, 142(6):721-729.

19. Yang $M$, Bao W, Zhang $X$, Kang $Y$, Haffty $B$, Zhang L: Short-term and long-term clinical outcomes of uncommon types of invasive breast cancer. Histopathology 2017, 71(6):874-886.

20. Mayo E, Llanos AA, Yi X, Duan SZ, Zhang $L$ : Prognostic value of tumour deposit and perineural invasion status in colorectal cancer patients: a SEERbased population study. Histopathology 2016, 69(2):230-238.

21. SEER Cause of Death Recode 1969+ (03/01/2018)

[https://web.archive.org/web/2019102 8030412/https://seer.cancer.gov/codre code/1969 d03012018/index.html]

22. Race Recode Changes: For the 19732005 SEER Research Data (November 2007 Submission) and Later Releases [https://web.archive.org/web/2019102 8023614/https://seer.cancer.gov/seerst at/variables/seer/race ethnicity/]
23. County attributes

[https://web.archive.org/web/2019102 8025023/https://seer.cancer.gov/seerst at/variables/countyattribs/]

24. Collaborative Stage Data Set: Prostate [https://web.archive.org/web/2019051 7115038/http://web2.facs.org/cstage0 205/prostate/Prostateschema.html]

25. Guo Y, Mao S, Zhang A, Zhang J, Wang L, Wang $R$, Zhang W, Zhang Z, Wu Y, Cao X et al: Survival Significance of Patients With Low Prostate-Specific Antigen and High-Grade Prostate Cancer After Radical Prostatectomy, External Beam Radiotherapy, or External Beam Radiotherapy With Brachytherapy. Frontiers in oncology 2019, 9:638.

26. Geographic Terms and Concepts Census Divisions and Census Regions [https://www.census.gov/geo/referenc e/gtc/gtc census divreg.html]

27. Radiation/Chemotherapy Databases (1975-2016)

[https://web.archive.org/save/https://s eer.cancer.gov/data/treatment.html]

28. Waldmann P: Approximate Bayesian neural networks in genomic prediction. Genet Sel Evol 2018, 50(1):70.

29. Agajanian S, Oluyemi O, Verkhivker GM: Integration of Random Forest Classifiers and Deep Convolutional Neural Networks for Classification and Biomolecular Modeling of Cancer Driver Mutations. Front Mol BiosCi 2019, 6:44.

30. Applications of Random Forest Algorithm

[https://web.archive.org/web/2019101 4125205/https://www.stata.com/meeti ng/canada18/slides/canada18 Zou.pdf]

31. Long JS, Freese J: Regression models for categorical dependent variables using Stata: Stata press; 2006.

32. Multinomial Logistic Regression Stata data analysis examples

[https://web.archive.org/web/2018101 0004634/https://stats.idre.ucla.edu/sta ta/dae/multinomiallogistic-regression/] 
33. Lin YT, Lee MT, Huang YC, Liu CK, Li YT, Chen M: Prediction of Recurrenceassociated Death from Localized Prostate Cancer with a Charlson Comorbidity Index-reinforced Machine Learning Model. Open medicine (Warsaw, Poland) 2019, 14:593-606.

34. Kleppe A, Albregtsen F, Vlatkovic L, Pradhan $M$, Nielsen $B$, Hveem TS, Askautrud HA, Kristensen GB, Nesbakken A, Trovik J et al: Chromatin organisation and cancer prognosis: a pan-cancer study. The Lancet Oncology 2018, 19(3):356-369.

35. Cruz JA, Wishart DS: Applications of machine learning in cancer prediction and prognosis. Cancer informatics 2007, 2:59-77.

36. Carmona R, Zakeri K, Green G, Hwang L, Gulaya S, Xu B, Verma R, Williamson CW, Triplett DP, Rose BS et al: Improved Method to Stratify Elderly Patients With Cancer at Risk for Competing Events. J Clin Oncol 2016, 34(11):12701277.

37. Song Y, Gao S, Tan W, Qiu Z, Zhou H, Zhao Y: Multiple Machine Learnings Revealed Similar Predictive Accuracy for Prognosis of PNETs from the Surveillance, Epidemiology, and End Result Database. Journal of Cancer 2018, 9(21):3971-3978.

38. van der Ploeg T, Nieboer D, Steyerberg EW: Modern modeling techniques had limited external validity in predicting mortality from traumatic brain injury. Journal of clinical epidemiology 2016, 78:83-89.

39. Zhang $\mathrm{Y}$, Yan L, Zeng J, Zhou H, Liu H, Yu G, Yao W, Chen K, Ye Z, Xu H: Pancancer analysis of clinical relevance of alternative splicing events in $\mathbf{3 1}$ human cancers. Oncogene 2019, 38(40):66786695.

40. Shen J, Wang L, Taylor JMG: Estimation of the optimal regime in treatment of prostate cancer recurrence from observational data using flexible weighting models. Biometrics 2017, 73(2):635-645.

41. Kourou K, Exarchos TP, Exarchos KP, Karamouzis MV, Fotiadis DI: Machine learning applications in cancer prognosis and prediction. Computational and structural biotechnology journal 2015, 13:8-17.

42. Fa B, Luo C, Tang Z, Yan Y, Zhang Y, Yu Z: Pathway-based biomarker identification with crosstalk analysis for robust prognosis prediction in hepatocellular carcinoma. EBioMedicine 2019, 44:250-260.

43. Hussain L, Ahmed A, Saeed S, Rathore $S$, Awan IA, Shah SA, Majid A, Idris A, Awan AA: Prostate cancer detection using machine learning techniques by employing combination of features extracting strategies. Cancer biomarkers : section A of Disease markers 2018, 21(2):393-413.

44. Agranoff $D$, Fernandez-Reyes $D$, Papadopoulos MC, Rojas SA, Herbster M, Loosemore A, Tarelli E, Sheldon J, Schwenk A, Pollok R et al: Identification of diagnostic markers for tuberculosis by proteomic fingerprinting of serum. Lancet (London, England) 2006, 368(9540):1012-1021.

45. Shameer K, Johnson KW, Glicksberg BS, Dudley JT, Sengupta PP: Machine learning in cardiovascular medicine: are we there yet? Heart 2018, 104(14):1156-1164.

46. Rodrigues $\mathrm{G}$, Warde $\mathrm{P}$, Pickles $\mathrm{T}$, Crook J, Brundage $M$, Souhami L, Lukka H, Genitourinary Radiation Oncologists of C: Pre-treatment risk stratification of prostate cancer patients: A critical review. Can Urol Assoc J 2012, 6(2):121127. 


\section{Figure Legends}

\section{Figure 1. Study flow.}

We randomized the patients into training and testing sets with similar sample-size in each group.We then tuned the random forest (RF) model, chose the best-fit RF model, and applied multinomial logistic (ML) model using training set. Using the ML and chosen RF models, we predicted 6-category causes of death among the patients in testing set. During the cross validation phase, we followed a similar protocol but with swapped datasets.

\section{Figure 2. Characteristics of random forest models.}

During primary validation process, prediction accuracies of random forest models varied by the corresponding numbers of variable and iteration (Heatmap graphs: A. categorical data encoding; B. Onehot data encoding). The random forest models provided computed relative importance-values for all included variables (C. and D. Relative-importance values of the top 10 variables in the chosen random forest models using categorical data encoding and one-hot data encoding, respectively). Note: *, Continuous variables were converted to 4-category variables by their respective quartiles; Dx, diagnosis; PSA, Prostate specific antigen; Education attainment defined as percent of residents with less than highschool graduate in the county; Person in poverty defined as percent of residents with income below $200 \%$ of poverty in the county.

\section{Figure 3. Summary of prediction accuracies by model and data type.}

In the tuning process and sensitivity analyses, we computed the validation accuracy of each random forest model by the numbers of variable and iteration $(n=240)$, and chose the one with the best accuracy as the final model. The error bars show 95\% confidence intervals of prediction accuracies in those models and data types during tuning process, except 3 models, whose $95 \%$ confidence intervals were calculated for the accuracy of a single model using binomial model (indicated by *). One-hot indicates one-hot encoding of the data; balanced set refers to the sensitivity analysis with training and testing sets that had balanced distribution of all variables.

Table 1. Baseline characteristics of included subjects

\begin{tabular}{|c|c|c|c|c|c|c|c|}
\hline & $\begin{array}{l}\text { Alive, } \\
n=29,611\end{array}$ & $\begin{array}{l}\text { CVD, } \\
n=5,448\end{array}$ & $\begin{array}{l}\text { Infection, } \\
\mathrm{n}=717\end{array}$ & $\begin{array}{l}\text { Non- } \\
\text { Prostate } \\
\text { cancer, } \\
n=3,681\end{array}$ & $\begin{array}{l}\text { Other } \\
\text { cause, } \\
n=5,800\end{array}$ & $\begin{array}{l}\text { Prostate } \\
\text { cancer, } \\
n=4,607\end{array}$ & $\begin{array}{l}\text { Total, } \\
n=49,864\end{array}$ \\
\hline Age (yr)ף & $63(50-77)$ & $\begin{array}{l}74 \text { (59- } \\
87)\end{array}$ & $\begin{array}{l}75(58- \\
88)\end{array}$ & $\begin{array}{l}70 \text { (56- } \\
83)\end{array}$ & $\begin{array}{l}73(58- \\
86)\end{array}$ & $\begin{array}{l}72(54- \\
88)\end{array}$ & $\begin{array}{l}67 \text { (51- } \\
83)\end{array}$ \\
\hline $\begin{array}{l}\text { Survival time } \\
\text { (mo)ף }\end{array}$ & $\begin{array}{l}146(131- \\
155)\end{array}$ & $\begin{array}{l}77(7- \\
143)\end{array}$ & $\begin{array}{l}78(6- \\
141)\end{array}$ & $\begin{array}{l}78(12- \\
141)\end{array}$ & $\begin{array}{l}82(11- \\
143)\end{array}$ & $\begin{array}{l}59(4- \\
137)\end{array}$ & $\begin{array}{l}117(16- \\
154)\end{array}$ \\
\hline \multicolumn{8}{|l|}{ Race } \\
\hline API & 1,453 & 210 & 43 & 172 & 268 & 195 & 2,341 \\
\hline
\end{tabular}




\begin{tabular}{|c|c|c|c|c|c|c|c|}
\hline (\%) & $(4.9)$ & (3.9) & (6.0) & $(4.7)$ & $(4.6)$ & $(4.2)$ & (4.7) \\
\hline Hispanic & 2,662 & 412 & 68 & 249 & 497 & 423 & 4,311 \\
\hline (\%) & $(9.0)$ & $(7.6)$ & (9.5) & (6.8) & (8.6) & $(9.2)$ & (8.7) \\
\hline NH Black & 3,830 & 865 & 143 & 553 & 807 & 812 & 7,010 \\
\hline (\%) & (12.9) & (15.9) & (19.9) & $(15.0)$ & (13.9) & $(17.6)$ & $(14.1)$ \\
\hline NH White & 21,093 & 3,920 & 461 & 2,690 & 4,189 & 3,147 & 35,500 \\
\hline (\%) & $(71.2)$ & $(72.0)$ & (64.3) & (73.1) & $(72.2)$ & (68.3) & $(71.2)$ \\
\hline Unknown/Other & 573 & 41 & $<15^{*}$ & 17 & 39 & 30 & 702 \\
\hline (\%) - & (1.9) & $(0.8)$ & & $(0.5)$ & $(0.7)$ & $(0.7)$ & (1.4) \\
\hline \multicolumn{8}{|l|}{ TNM6 T category } \\
\hline $\mathrm{T} 1 / 2$ & 26,641 & 4,873 & 635 & 3,245 & 5,201 & 2,917 & 43,512 \\
\hline (\%) & $(90.0)$ & (89.5) & (88.6) & $(88.2)$ & (89.7) & (63.3) & (87.3) \\
\hline $\mathrm{T} 3 / 4$ & 2,543 & 278 & 40 & 281 & 329 & 890 & 4,361 \\
\hline (\%) & $(8.6)$ & (5.1) & $(5.6)$ & $(7.6)$ & (5.7) & (19.3) & (8.8) \\
\hline Unknown/Other & 427 & 297 & 42 & 155 & 270 & 800 & 1,991 \\
\hline (\%) - & $(1.4)$ & (5.5) & (5.9) & $(4.2)$ & (4.7) & $(17.4)$ & $(4.0)$ \\
\hline \multicolumn{8}{|l|}{ TNM6 N category } \\
\hline 0 & 28,140 & 4,850 & 631 & 3,354 & 5,226 & 3,057 & 45,258 \\
\hline (\%) & $(94.7)$ & (88.3) & (87.2) & $(90.6)$ & (89.4) & $(65.2)$ & $(90.2)$ \\
\hline 1 & 283 & 64 & $<15^{*}$ & 60 & 55 & 357 & 830 \\
\hline (\%) & (1.0) & $(1.2)$ & & $(1.6)$ & (0.9) & (7.6) & (1.7) \\
\hline Unknown/Other & 1,307 & 579 & 82 & 289 & 566 & 1,272 & 4,095 \\
\hline (\%) - & $(4.4)$ & $(10.5)$ & (11.3) & (7.8) & (9.7) & (27.1) & (8.2) \\
\hline \multicolumn{8}{|l|}{ TNM6 M category } \\
\hline 0 & 28,615 & 4,911 & 648 & 3,389 & 5,291 & 2,794 & 45,648 \\
\hline (\%) & $(96.3)$ & (89.4) & (89.5) & (91.5) & (90.5) & $(59.6)$ & (91.0) \\
\hline 1 & 160 & 182 & 25 & 120 & 174 & 1,363 & 2,024 \\
\hline (\%) & $(0.5)$ & (3.3) & (3.5) & (3.2) & (3.0) & (29.1) & (4.0) \\
\hline Unknown/Other & 955 & 400 & 51 & 194 & 382 & 529 & 2,511 \\
\hline (\%) - & (3.2) & (7.3) & (7.0) & $(5.2)$ & (6.5) & (11.3) & (5.0) \\
\hline \multicolumn{8}{|l|}{ AJCC6 staging } \\
\hline 1 & 47 & 21 & $<15^{*}$ & $<15^{*}$ & 26 & $<15^{*}$ & 110 \\
\hline (\%) & $(0.2)$ & $(0.4)$ & & & $(0.5)$ & & $(0.2)$ \\
\hline 2 & 25476 & 4459 & 574 & 3013 & 4785 & 2054 & 40361 \\
\hline (\%) & $(86.0)$ & (81.9) & (80.1) & (81.9) & (82.5) & $(44.6)$ & (80.9) \\
\hline 3 & 2110 & 173 & 19 & 200 & 216 & 328 & 3046 \\
\hline (\%) & (7.1) & (3.2) & (2.7) & $(5.4)$ & (3.7) & $(7.1)$ & (6.1) \\
\hline 4 & 607 & 252 & 38 & 200 & 240 & 1595 & 2932 \\
\hline (\%) - & $(2.1)$ & (4.6) & (5.3) & $(5.4)$ & (4.1) & $(34.6)$ & (5.9) \\
\hline Unknown/Other & 1371 & 543 & 81 & 258 & 533 & 629 & 3415 \\
\hline (\%) - & $(4.6)$ & $(10.0)$ & (11.3) & (7.0) & (9.2) & (13.7) & (6.9) \\
\hline \multicolumn{8}{|l|}{ Chemotherapy } \\
\hline None/Unknown & 29,617 & 5,472 & 720 & 3,671 & 5,821 & 4,516 & 49,817 \\
\hline (\%) - & (99.6) & (99.6) & (99.5) & (99.1) & (99.6) & (96.4) & (99.3) \\
\hline
\end{tabular}




\begin{tabular}{|c|c|c|c|c|c|c|c|}
\hline $\begin{array}{l}\text { Received } \\
(\%)\end{array}$ & $\begin{array}{r}113 \\
(0.4)\end{array}$ & $\begin{array}{r}21 \\
(0.4)\end{array}$ & $<15^{*}$ & $\begin{array}{r}32 \\
(0.9)\end{array}$ & $\begin{array}{r}26 \\
(0.4)\end{array}$ & $\begin{array}{r}170 \\
(3.6)\end{array}$ & $\begin{array}{r}366 \\
(0.7)\end{array}$ \\
\hline \multicolumn{8}{|l|}{ Radiotherapy } \\
\hline None/Unknown & 18,450 & 3,364 & 446 & 2,094 & 3,537 & 3,257 & 31,148 \\
\hline (\%) & $(62.1)$ & $(61.2)$ & $(61.6)$ & $(56.6)$ & $(60.5)$ & (69.5) & $(62.1)$ \\
\hline Received & 11,280 & 2,129 & 278 & 1,609 & 2,310 & 1,429 & 19,035 \\
\hline (\%) & (37.9) & (38.8) & $(38.4)$ & (43.5) & (39.5) & $(30.5)$ & (37.9) \\
\hline \multicolumn{8}{|l|}{ Surgery } \\
\hline Local Excision & 1,093 & 599 & 72 & 270 & 657 & 483 & 3,174 \\
\hline (\%) & (3.7) & $(11.0)$ & (10.0) & (7.3) & (11.3) & $(10.5)$ & (6.4) \\
\hline No surgery & 15,142 & 4,261 & 578 & 2,649 & 4,413 & 3,666 & 30,709 \\
\hline (\%) & $(51.1)$ & $(78.2)$ & $(80.6)$ & $(72.0)$ & (76.1) & (79.6) & (61.6) \\
\hline Prostatectomy & 13,376 & 588 & 67 & 762 & 730 & 458 & 15,981 \\
\hline$(\%)$ & $(45.2)$ & $(10.8)$ & (9.3) & $(20.7)$ & $(12.6)$ & (9.9) & (32.1) \\
\hline \multicolumn{8}{|c|}{ Rural-urban continuum $2003 \S$} \\
\hline Metro & 26,709 & 4,758 & 635 & 3,178 & 4,958 & 4,039 & 44,277 \\
\hline$(\%)$ & $(89.8)$ & $(86.6)$ & $(87.7)$ & $(85.8)$ & $(84.8)$ & $(86.2)$ & $(88.2)$ \\
\hline Non-Metro & 3,021 & 735 & 89 & 525 & 889 & 647 & 5,906 \\
\hline 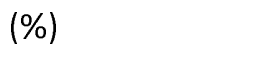 & $(10.2)$ & $(13.4)$ & $(12.3)$ & $(14.2)$ & $(15.2)$ & $(13.8)$ & $(11.8)$ \\
\hline \multicolumn{8}{|l|}{ Census region } \\
\hline Midwest & 2,946 & 658 & 77 & 399 & 613 & 429 & 5,122 \\
\hline$(\%)$ & $(10.0)$ & $(12.1)$ & $(10.7)$ & $(10.8)$ & $(10.6)$ & (9.3) & $(10.3)$ \\
\hline Northeast & 4,797 & 882 & 123 & 631 & 874 & 721 & 8,028 \\
\hline$(\%)$ & $(16.2)$ & $(16.2)$ & $(17.2)$ & $(17.1)$ & $(15.1)$ & $(15.7)$ & $(16.1)$ \\
\hline South & 5,573 & 1,140 & 176 & 843 & 1,393 & 1,009 & 10,134 \\
\hline$(\%)$ & $(18.8)$ & (20.9) & $(24.6)$ & (22.9) & $(24.0)$ & (21.9) & $(20.3)$ \\
\hline West & 16,295 & 2,768 & 341 & 1,808 & 2,920 & 2,448 & 26,580 \\
\hline 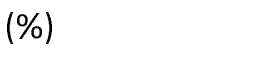 & $(55.0)$ & $(50.8)$ & $(47.6)$ & $(49.1)$ & $(50.3)$ & $(53.1)$ & $(53.3)$ \\
\hline \multicolumn{8}{|c|}{ Percent of education attainment, quartile§ } \\
\hline Q1, <15.08 & 8,001 & 1,200 & 140 & 836 & 1,339 & 1,029 & 12,545 \\
\hline (\%) & (26.9) & (21.9) & (19.3) & $(22.6)$ & (22.9) & $(22.0)$ & $(25.0)$ \\
\hline Q2, 15.09-18.15 & 7,538 & 1,287 & 182 & 898 & 1,448 & 1,193 & 12,546 \\
\hline (\%) & $(25.4)$ & $(23.4)$ & $(25.1)$ & $(24.3)$ & $(24.8)$ & $(25.5)$ & $(25.0)$ \\
\hline Q3, 18.17-25.79 & 7,236 & 1,420 & 189 & 997 & 1,492 & 1,212 & 12,546 \\
\hline (\%) & $(24.3)$ & (25.9) & (26.1) & (26.9) & $(25.5)$ & (25.9) & $(25.0)$ \\
\hline$Q 4,>50.77$ & 6,955 & 1,586 & 213 & 972 & 1,568 & 1,252 & 12,546 \\
\hline (\%) & $(23.4)$ & (28.9) & $(29.4)$ & $(26.3)$ & $(26.8)$ & $(26.7)$ & $(25.0)$ \\
\hline \multicolumn{8}{|c|}{ Percent of persons in poverty, quartile§ } \\
\hline $\mathrm{Q} 1,<21.18$ & 8,034 & 1,210 & 160 & 865 & 1,305 & 1,044 & 12,618 \\
\hline (\%) & $(27.0)$ & $(22.0)$ & $(22.1)$ & $(23.4)$ & $(22.3)$ & (22.3) & $(25.1)$ \\
\hline Q2, 21.33-29.81 & 7,655 & 1,258 & 152 & 929 & 1,364 & 1,129 & 12,487 \\
\hline (\%) & $(25.8)$ & $(22.9)$ & $(21.0)$ & $(25.1)$ & $(23.3)$ & $(24.1)$ & (24.9) \\
\hline Q3, 29.86-37.36 & 7,276 & 1,493 & 220 & 986 & 1,662 & 1,256 & 12,893 \\
\hline 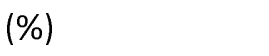 & $(24.5)$ & $(27.2)$ & $(30.4)$ & $(26.6)$ & $(28.4)$ & $(26.8)$ & $(25.7)$ \\
\hline
\end{tabular}




\begin{tabular}{|c|c|c|c|c|c|c|c|}
\hline $\begin{array}{l}\text { Q4, >67.40 } \\
\text { (\%) }\end{array}$ & $\begin{array}{r}6,765 \\
(22.8)\end{array}$ & $\begin{array}{l}1,532 \\
(27.9)\end{array}$ & $\begin{array}{r}192 \\
(26.5)\end{array}$ & $\begin{array}{r}923 \\
(24.9)\end{array}$ & $\begin{array}{l}1,516 \\
(25.9)\end{array}$ & $\begin{array}{l}1,257 \\
(26.8)\end{array}$ & $\begin{array}{r}12,185 \\
(24.3)\end{array}$ \\
\hline \multicolumn{8}{|c|}{ Percent of foreign-born residents, quartile§ } \\
\hline $\mathrm{Q} 1,<5.95$ & 6,864 & 1,467 & 188 & 1,041 & 1,747 & 1,254 & 12,561 \\
\hline (\%) & $(23.1)$ & $(26.7)$ & $(26.0)$ & $(28.1)$ & (29.9) & $(26.8)$ & $(25.0)$ \\
\hline Q2, 5.98-15.22 & 7,739 & 1,399 & 172 & 922 & 1,498 & 1,157 & 12,887 \\
\hline (\%) & $(26.0)$ & $(25.5)$ & $(23.8)$ & (24.9) & $(25.6)$ & $(24.7)$ & (25.7) \\
\hline Q3, 15.45-21.55 & 7,412 & 1,257 & 179 & 866 & 1,342 & 1,171 & 12,227 \\
\hline (\%) & $(24.9)$ & (22.9) & $(24.7)$ & $(23.4)$ & $(23.0)$ & $(25.0)$ & $(24.4)$ \\
\hline $\mathrm{Q} 4,>38.52$ & 7,715 & 1,370 & 185 & 874 & 1,260 & 1,104 & 12,508 \\
\hline (\%) & $(26.0)$ & (24.9) & $(25.6)$ & $(23.6)$ & $(21.6)$ & $(23.6)$ & (24.9) \\
\hline \multicolumn{8}{|c|}{ Confirmation method of diagnosis } \\
\hline Microscopic & 29,628 & 5,321 & 697 & 3,652 & 5,688 & 4,223 & 49,209 \\
\hline 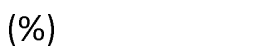 & (99.7) & (96.9) & $(96.3)$ & (98.6) & (97.3) & $(90.1)$ & (98.1) \\
\hline \multicolumn{8}{|l|}{ Radiologic and } \\
\hline clinic & 40 & 122 & 21 & 43 & 104 & 285 & 615 \\
\hline$(\%)$ & $(0.1)$ & $(2.2)$ & (2.9) & (1.2) & (1.8) & $(6.1)$ & $(1.2)$ \\
\hline Unknown/Other & 62 & 50 & $<15^{*}$ & $<15^{*}$ & 55 & 178 & 359 \\
\hline 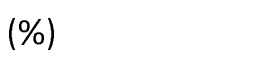 & $(0.2)$ & (0.9) & & & (0.9) & (3.8) & $(0.7)$ \\
\hline \multicolumn{8}{|c|}{ PSA, quartiles (ng/ml) } \\
\hline$<4.9$ & 8,360 & 765 & 88 & 665 & 874 & 367 & 11,119 \\
\hline (\%) & $(28.2)$ & $(14.0)$ & $(12.3)$ & $(18.1)$ & $(15.1)$ & (8.0) & (22.3) \\
\hline $5.0-6.8$ & 7,406 & 829 & 108 & 735 & 1,023 & 337 & 10,438 \\
\hline (\%) & $(25.0)$ & $(15.2)$ & (15.1) & $(20.0)$ & $(17.6)$ & (7.3) & (20.9) \\
\hline $6.9-11.3$ & 6,331 & 1,199 & 157 & 804 & 1,239 & 580 & 10,310 \\
\hline$(\%)$ & $(21.4)$ & $(22.0)$ & (21.9) & $(21.8)$ & $(21.4)$ & $(12.6)$ & $(20.7)$ \\
\hline $11.3+$ & 4,081 & 1,487 & 216 & 887 & 1,494 & 2,331 & 10,496 \\
\hline 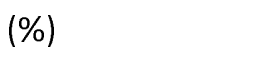 & $(13.8)$ & $(27.3)$ & (30.1) & (24.1) & $(25.8)$ & $(50.6)$ & (21.1) \\
\hline Unknown/Other & 3,433 & 1,168 & 148 & 590 & 1,170 & 992 & 7,501 \\
\hline (\%) & $(11.6)$ & $(21.4)$ & $(20.6)$ & $(16.0)$ & $(20.2)$ & (21.5) & $(15.0)$ \\
\hline \multicolumn{8}{|l|}{ Gleason score } \\
\hline 5 & $<15^{*}$ & $<15^{*}$ & $<15^{*}$ & $<15^{*}$ & $<15^{*}$ & $<15^{*}$ & 15 \\
\hline 6 & 264 & 37 & $<15^{*}$ & 21 & 26 & 19 & 374 \\
\hline (\%) & (0.9) & (0.7) & & $(0.6)$ & (0.5) & $(0.4)$ & (0.8) \\
\hline 7 & 219 & 29 & $<15^{*}$ & 22 & 28 & 32 & 335 \\
\hline (\%) & (0.7) & (0.5) & & $(0.6)$ & $(0.5)$ & (0.7) & $(0.7)$ \\
\hline 8 & 36 & 15 & $<15^{*}$ & $<15^{*}$ & $<15^{*}$ & 20 & 94 \\
\hline (\%) & $(0.1)$ & $(0.3)$ & & & & $(0.4)$ & $(0.2)$ \\
\hline 9 & $<15^{*}$ & $<15^{*}$ & $<15^{*}$ & $<15^{*}$ & $<15^{*}$ & $<15^{*}$ & 65 \\
\hline (\%) & & & & & & & $(0.1)$ \\
\hline 10 & $<15^{*}$ & $<15^{*}$ & $<15^{*}$ & $<15^{*}$ & $<15^{*}$ & $<15^{*}$ & $<15^{*}$ \\
\hline $\begin{array}{l}\text { Unknown/Other } \\
\text { (\%) }\end{array}$ & $\begin{array}{r}29,069 \\
(98.2)\end{array}$ & $\begin{array}{l}5,358 \\
(98.4)\end{array}$ & $\begin{array}{r}701 \\
(97.8)\end{array}$ & $\begin{array}{l}3,624 \\
(98.5)\end{array}$ & $\begin{array}{l}5,728 \\
(98.8)\end{array}$ & $\begin{array}{l}4,493 \\
(97.5)\end{array}$ & $\begin{array}{r}48,973 \\
(98.2)\end{array}$ \\
\hline
\end{tabular}


Note: AJCC, $6^{\text {th }}$ edition clinical staging of the American Joint Commission on Cancer; TNM6, $6^{\text {th }}$ edition Tumor, node and metastasis staging manual of the American Joint Commission on Cancer; API, Asian Pacific Islanders; NH, Non-Hispanic; CVD, cardiovascular disease; PSA, Prostate specific antigen; *, statistically suppressed; I 95\% confidence intervals in parenthesis; $§$, County attributes of Year 2000; Education attainment defined as percent of residents with less than high-school graduate in the county; Person in poverty defined as percent of residents with income below $200 \%$ of poverty in the county.

Table 2. Prediction accuracy for long-term 6-category causes of death among the patients with prostate cancer diagnosis in 2004 (follow up through Dec. 2016)

\begin{tabular}{|c|c|c|c|c|c|c|c|}
\hline Predicted classes & $\begin{array}{l}\text { Alive, } \\
\mathrm{n}=14,746\end{array}$ & $\begin{array}{l}\text { CVD, } \\
n=2,689\end{array}$ & $\begin{array}{l}\text { Infection, } \\
n=371\end{array}$ & $\begin{array}{l}\text { Non- } \\
\text { Prostate } \\
\text { cancer, } \\
n=1,873\end{array}$ & $\begin{array}{l}\text { Other } \\
\text { cause, } \\
n=2,897\end{array}$ & $\begin{array}{l}\text { Prostate } \\
\text { cancer, } \\
n=2,288\end{array}$ & $\begin{array}{l}\text { Total, } \\
n=24,864\end{array}$ \\
\hline \multicolumn{8}{|l|}{ Random forest model } \\
\hline Alive, $\%$ & $87.70 *$ & 52.73 & 52.29 & 67.49 & 55.82 & 39.9 & 73.75 \\
\hline CVD, $\%$ & 3.79 & $15.88^{*}$ & 15.90 & 10.04 & 15.08 & 8.92 & 7.54 \\
\hline Infection, \% & 0.21 & 0.67 & $0.27^{*}$ & 0.32 & 0.69 & 0.31 & 0.33 \\
\hline Non-Prostate cancer, \% & 1.94 & 3.35 & 2.96 & $2.94^{*}$ & 3.11 & 3.23 & 2.44 \\
\hline Other cause, \% & 3.82 & 17.44 & 16.44 & 10.62 & $15.05^{*}$ & 10.01 & 7.87 \\
\hline Prostate cancer, $\%$ & 2.54 & 9.93 & 12.13 & 8.60 & 10.25 & $37.63^{*}$ & 8.06 \\
\hline \multicolumn{8}{|l|}{ Multinomial model } \\
\hline Alive, \% & $82.63^{*}$ & 33.51 & 31.27 & 51.84 & 37.04 & 32.87 & 64.34 \\
\hline NA, \% & 17.37 & 66.49 & 68.73 & 48.16 & 62.96 & 67.13 & 35.66 \\
\hline
\end{tabular}

Note: CVD, cardiovascular disease; NA, not available; *, correct prediction. 


\begin{tabular}{|c|c|c|c|c|}
\hline $\begin{array}{c}\text { Included } 49,864 \\
\text { prostate cancer cases }\end{array}$ & \multirow{2}{*}{$\begin{array}{l}\text { Randomly divided cases into } \\
\text { the Training set }(n=25,000) \\
\text { and Testing set }(n=24,864)\end{array}$} & \multirow{2}{*}{$\begin{array}{c}\text { Training phase } \\
\begin{array}{c}\text { Cross validation } \\
\text { phase }\end{array} \\
\end{array}$} & $\begin{array}{l}\text { Train and tune the RF } \\
\text { or apply ML models } \\
\text { using the Training set }\end{array}$ & $\begin{array}{l}\text { Predict outcomes } \\
\text { using the Testing set }\end{array}$ \\
\hline $\begin{array}{l}\text { of SEER18 diagnosed } \\
\text { in 2004, with follow } \\
\text { up through Dec. } 2016\end{array}$ & & & $\begin{array}{l}\text { Train and tune the RF } \\
\text { or apply ML models } \\
\text { using the Testing set }\end{array}$ & $\begin{array}{l}\text { Predict outcomes } \\
\text { using the Training set }\end{array}$ \\
\hline
\end{tabular}




\section{$\Delta \&$. Tuning the random forest models using categorical-encoded data}

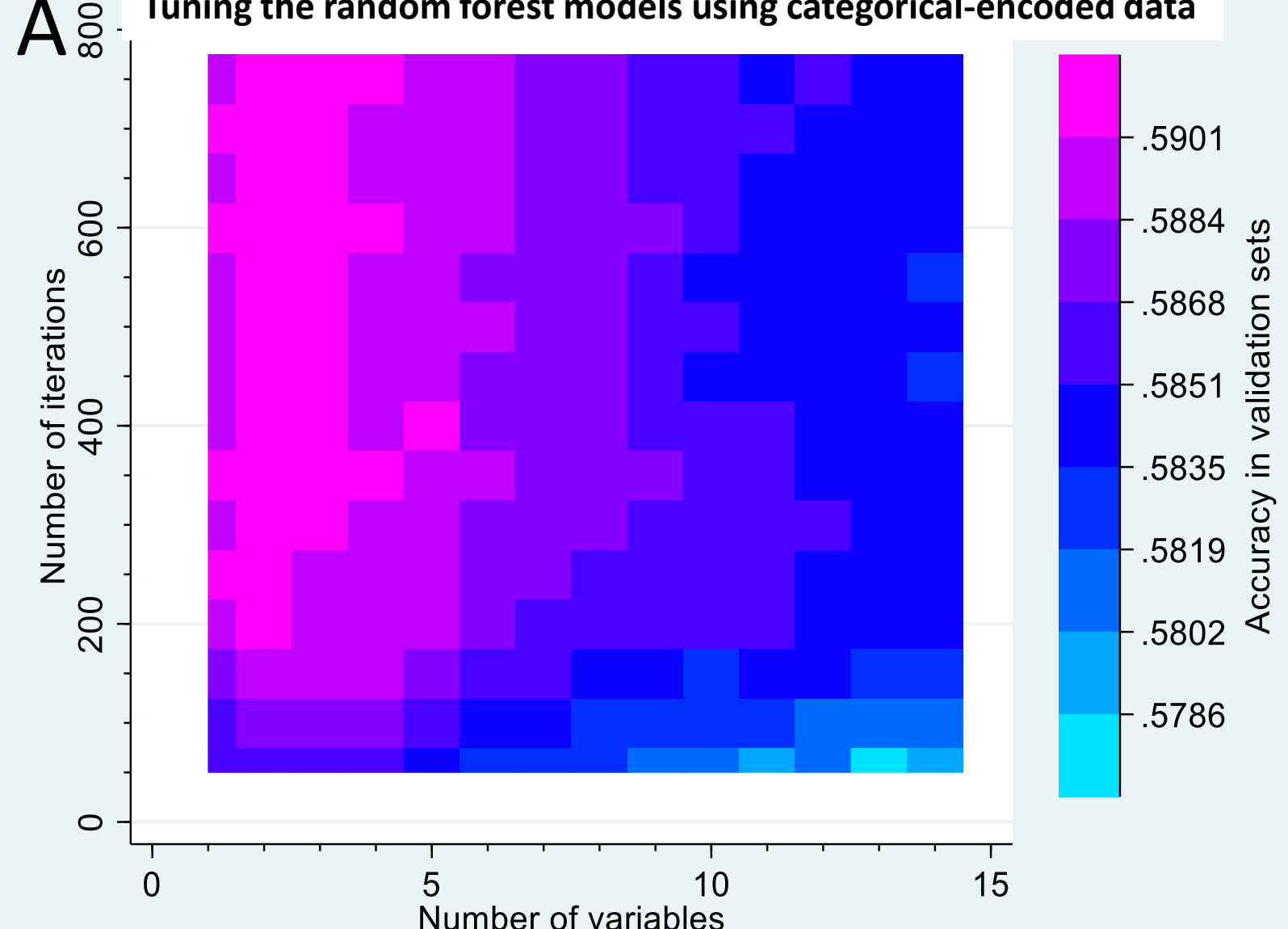


$B \&+$ Tuning the random forest models using one-hot encoded data

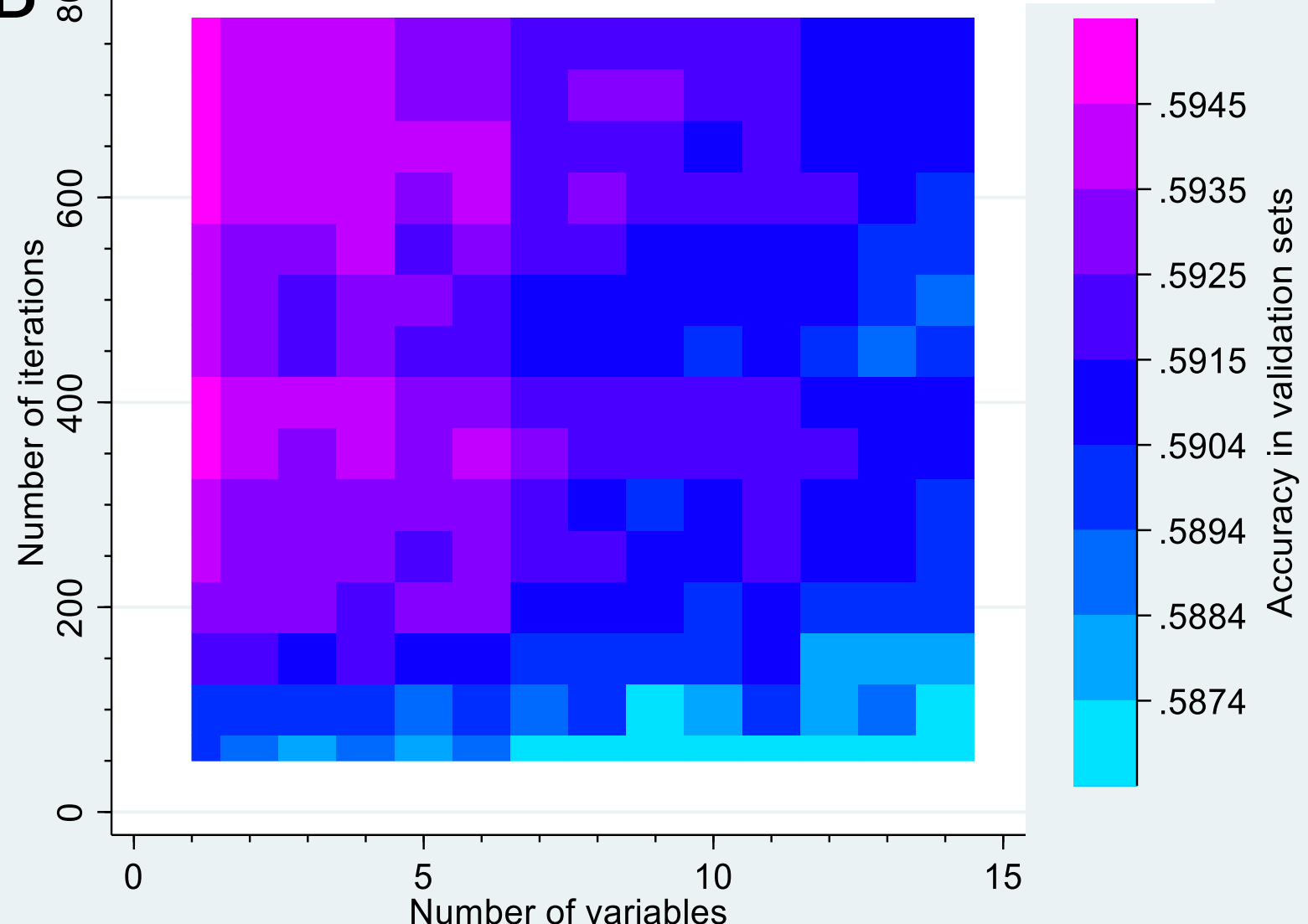




\section{Relative importance in the model using categorical-encoded data}

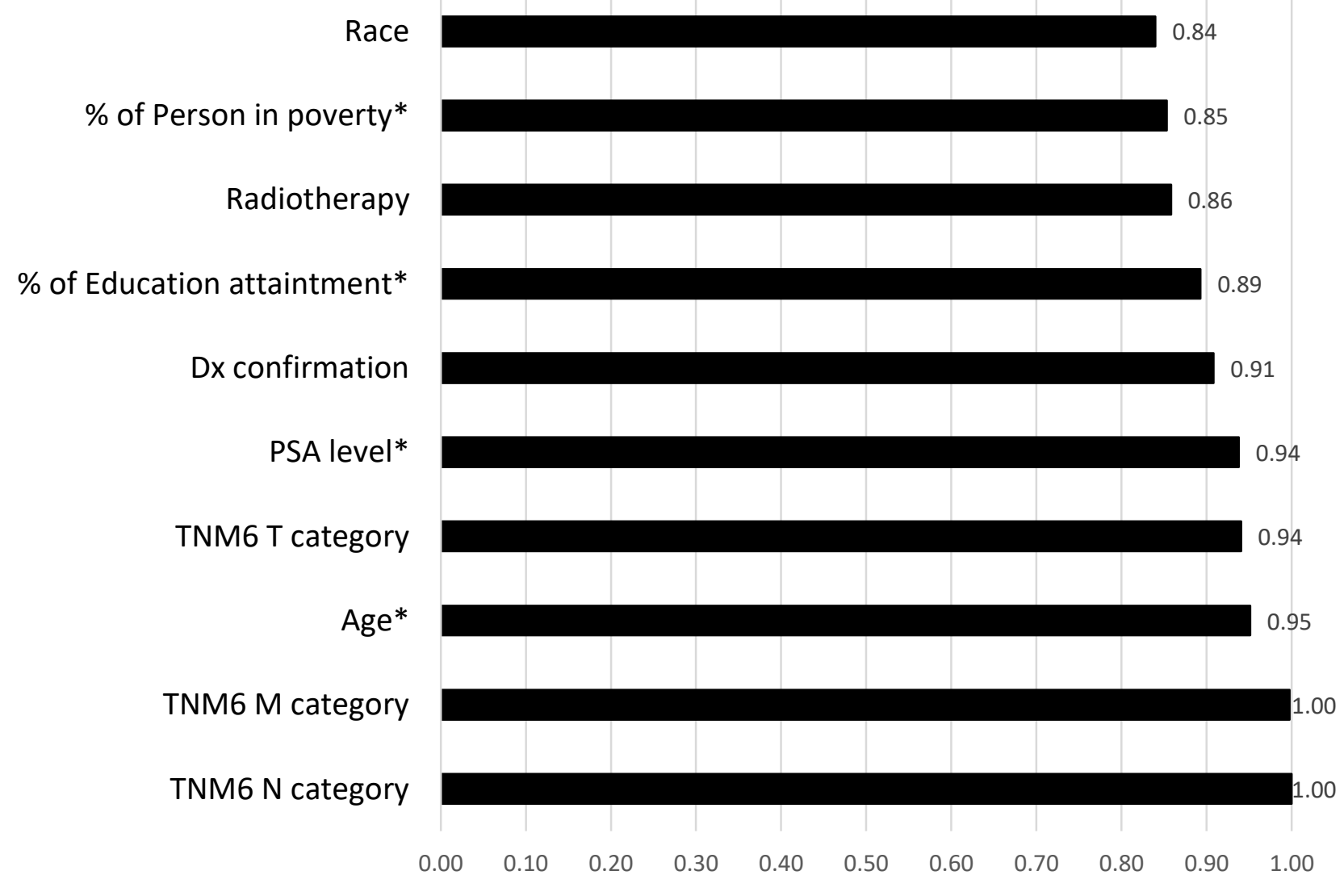


Relative importance in the model using one-hot encoded data

Dx Confirmation, Microscopic

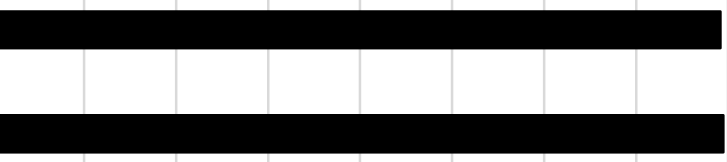

TNM6 T1/2

Age Quartile 3 (67-74yr)

PSA Quartile $4(11.3+\mathrm{ng} / \mathrm{mL})$

TNM6 M category, Others/Unknown

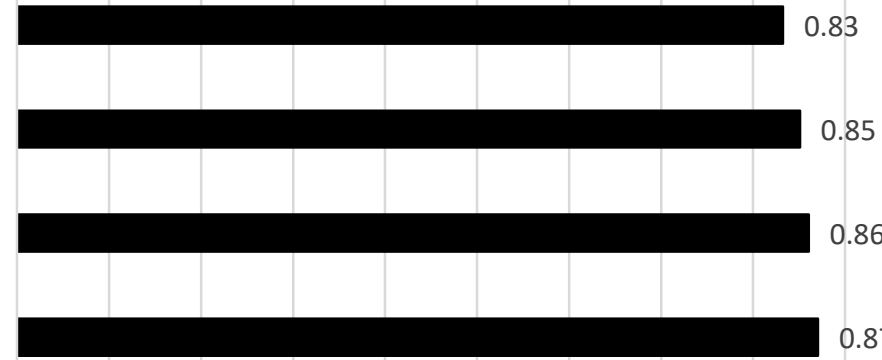

Radiotherapy

TNM6 M0

TNM6 T category, Others/Unknown

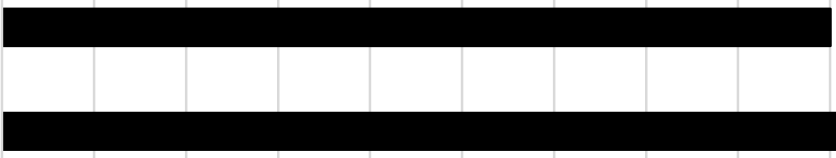

Age Quartile 4 (75+ yr) 


\section{Prediction Accuracy}

Deceased or longlived patients, one-hot data Multinomial logistic model, categorical data* Multinomial logistic model, one-hot data* Development phase, categorical data Cross validation, categorical data Development phase, one-hot data excluding GS Development phase, one-hot data Cross validation, one-hot data

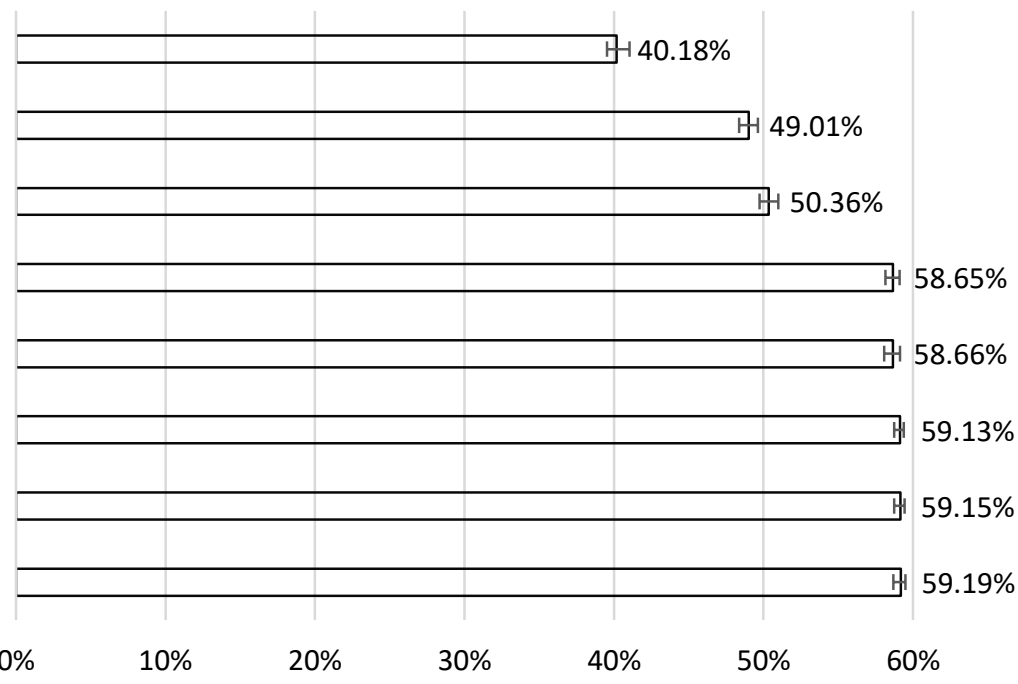

